\title{
SOBRE LA NATURALEZA JURÍDICA DE LA CARTA DE DERECHOS FUNDAMENTALES DE LA UNIÓN EUROPEA
}

ÁNGEL RODRÍGUEZ DÍAZ

Catedrático de Derecho Constitucional

Universidad de Extremadura 


\section{SUMARIO}

I. Introducción. - II. El debate politico sobre la naturaleza de la Carta. - III. La NATURALEZA JURÍDICA.- IV. El EFECTO VINCULANTE.- V. LA INCOPORACIÓN A LOS TRATADOS.- VI. UNA REFLEXIÓN FINAL.- VII. FUENTES Y REFERENCIAS.- 


\title{
SOBRE LA NATURALEZA JURÍDICA DE LA CARTA DE DERECHOS FUNDAMENTALES DE LA UNIÓN EUROPEA ${ }^{1}$
}

POR

\author{
ÁNGEL RODRÍGUEZ \\ Catedrático de Derecho Constitucional \\ Universidad de Extremadura
}

\section{INTRODUCCIÓN}

Desde diciembre de 2000 la Unión Europea posee ya, tras un largo e intenso debate jurídico y político, su propia Declaración de Derechos, la Carta de Derechos Fundamentales de la Unión Europea (citada en adelante como "la Carta" o con las siglas CDFUE), en la que algunos han querido incluso ver el embrión de lo que podría ser la futura Constitución de Europa. La Carta, sin embargo, no ha sido, como se sabe, formalmente incorporada a los tratados comunitarios (al menos por ahora), por lo que no han sido pocas las voces que se han alzado para denunciar que la misma no vendría a ser sino una más entre las

1 Este trabajo pretende sumarse a la celebración del vigésimo aniversario de la Facultad de Derecho de la Universidad de Málaga (1980-2000), a la que, a lo largo de diecisiete años, he estado ligado como profesor. Por circunstancias diversas, no imputables en ningún caso a sus promotores, no pudo en su momento ser incluido en el correspondiente libro conmemorativo. 
varias "declaraciones solemnes" con las que, a lo largo de su historia, la Unión ha tratado de resolver la espinosa cuestión de la ausencia en su ordenamiento de una norma que protegiera, al más alto nivel, los derechos fundamentales de sus ciudadanos.

Aun aceptando, en parte, estos razonamientos, se argumenta en el presente trabajo que la vinculación jurídica que pueden llegar a generar normas como la CDFUE depende más de su eficacia social y del uso que de la misma hagan los diferentes agentes y operadores jurídicos que de la definición que de la misma se haga en el propio texto legal, que, con todo, presenta algunas potencialidades que propiciarán probablemente en el futuro, del modo que se verá, la aplicación de la Carta por parte del TJCE y de los tribunales nacionales como norma jurídica vinculante.

\section{EL DEBATE POLÍTICO SOBRE LA NATURALEZA DE LA CARTA}

La existencia de similitudes entre el proceso de aprobación de la CDFUE y el de algunos textos constitucionales europeos, lejos de sorprender, confirma la imagen que la Carta ha tenido desde el primer momento - más adelante veremos si jurídicamente acertada o no- de Declaración Constitucional de Derechos. No sólo su existencia ha contribuido a animar, en el campo que le es propio, la vieja polémica sobre si Europa debe o no tener una Constitución, y sobre si, en todo caso, la Carta formaría parte de ella; otros factores han apuntalado esta visión: de una parte, la acentuada participación de la sociedad civil en su gestación, hasta el punto de que se llegó a equiparar la Convención que la redactó con unos nuevos Estados Generales de Europa (con las reminiscencias revolucionarias de todo tipo que trae consigo la expresión); de otra, las controversias entre los distintos órganos que debían participar en su aprobación final, que, con las particularidades propias de la complejidad institucional comunitaria, no dejaban de recordar las tensiones entre parlamentos $y$ monarcas en cuyo seno se gestaron buena parte de las Constituciones que las naciones europeas han tenido a lo largo de su historia.

Las reminiscencias constitucionales de la Carta se completan con el debate sobre el carácter bien vinculante bien meramente programático, de su texto, cuestión de nuevo muy familiar para el constitucionalista, pues, como las anteriores, no ha dejado de estar presente (también mutatis mutandis) en buena parte de los textos constitucionales 
del continente. Es a esta última cuestión a la que se refiere la polémica sobre la naturaleza jurídica de la Carta, cuyo planteamiento se remonta a los informes académicos que la precedieron y que justificaron su adopción.

La cumbre de Colonia, de junio de 1999, que dio formalmente curso al mandato para redactar una Carta de Derechos, dejó expresamente abierta la cuestión de su fuerza vinculante, especificando tan sólo el Consejo Europeo en sus conclusiones que, una vez elaborada la Carta, "habrá que estudiar si debe incorporarse (..) a los Tratados y, en caso afirmativo, de qué modo ha de hacerse". Tampoco se tomó ninguna decisión al respecto seis meses más tarde, cuando el Consejo Europeo de Tampere dispuso la composición y el método de trabajo de la Convención que habría de redactarla, pero sin decidir aún sobre su incorporación o no al derecho originario.

De modo que, al inicio del 2000 , la Convención redactora comenzó sus trabajos sin que se hubiera despejado previamente este interrogante, dando lugar así a una situación de incertidumbre que se mantuvo durante todo el año, hasta la proclamación solemne de la Carta llevada a cabo en Niza en el mes de diciembre.

De hecho, la Convención decidió expresamente, para atenerse al mandato conferido por el Consejo, concentrarse sólo en la redacción del articulado, sin debatir en su seno la cuestión de la incorporación. Ahora bien, dada la imposibilidad de avanzar en los trabajos de redacción sin despejar previamente cuál sería la naturaleza jurídica del texto que se estaba discutiendo, la propia Convención tomó una importante decisión de indudable alcance jurídico: debatir el articulado de la Carta "como sin la misma fuera a incorporarse a los tratados. Es decir, aun sin pronunciarse sobre el particular, redactar un texto con las características apropiadas para que, llegado el caso, pudiera decidirse su incorporación al derecho originario sin necesidad de introducir ninguna modificación. El resultado de esta particular técnica no fue sólo el desbloqueo de los trabajos de la Convención, sino la aprobación de un texto articulado conscientemente redactado para hacer posible su eventual aplicación como norma jurídica vinculante.

Debido a las limitaciones impuestas por el mandato del Consejo, la Convención redactora de la Carta no pudo pues pronunciarse sobre su carácter vinculante (aunque la técnica del "como si" puede sin duda interpretarse como un pronunciamiento implícito a favor de la incorporación), pero sí lo hicieron el resto de las instituciones comunitarias, 
mostrándose siempre, de forma más o menos directa, y prácticamente desde el comienzo mismo de los debates, a favor de la plena incorporación de la Carta al derecho originario.

Una primera toma de postura tuvo lugar en el Parlamento Europeo, cuyos delegados constituían un cuarto de los miembros de la Convención, y que había emitido ya previamente varias resoluciones apoyando la iniciativa de redactar una Carta de Derechos. El Parlamento se pronunció sobre el particular el 16 de marzo, anunciando su decisión, tomada en sesión plenaria, de "manifestar con toda claridad" que subordinaba "en un grado muy elevado" la aprobación definitiva de la Carta al cumplimiento de una serie de condiciones, entre las que incluía algunas reivindicaciones sobre la participación del propio Parlamento en su proceso de elaboración y, por lo que hacia a la naturaleza jurídica de la misma, que ésta debía "adquirir pleno carácter vinculante mediante su inclusión en el Tratado de la Unión Europea».

A medida que se acercaba el plazo en el que la Convención debía finalizar su trabajo - que debía estar listo para la cumbre informal de Biarritz, a celebrar en octubre- el resto de las instituciones comunitarias hicieron también público su parecer. El 20 de septiembre se aprobaron sendas resoluciones del Comité Económico y Social y del Comité de las Regiones. Para el primero, la naturaleza jurídica de la Carta debía implicar dotarle de "fuerza vinculante", y si bien la misma no tenía por qué asegurarse necesariamente mediante su incorporación a los tratados, ésta se postulaba como la mejor solución a adoptar. El Comité de las Regiones, al tiempo que recomendaba algunas modificaciones en cuanto a su contenido, se pronunció igualmente a favor de una Carta jurídicamente vinculante e integrada en los Tratados.

En este mismo mes de septiembre, la Comisión hizo también pública su opinión. En su comunicado, dirigido a uponer de relieve (..) cuestiones políticas e institucionales que (..) revisten especial importancia, en particular en lo que se refiere a la naturaleza de la Carta", saludaba la opción hecha por la Convención de trabajar sobre ésta "como si debiera incorporarse posteriormente a los Tratados comunitarios con fuerza jurídica vinculante», pronunciándose, si bien sólo indirectamente, a favor de esta opción, resaltando que

«la incorporación de la Carta a los Tratados permitiría poner remedio a algunas insuficiencias del sistema actual de protección de los derechos fundamentales en la Unión. Este sistema, en efecto, se caracteriza por una protección indirecta a través de los principios generales del Derecho comunitario, protección esencialmente pre- 
toriana fijada por la jurisprudencia al albur de los asuntos presentados ante las jurisdicciones, protección que no es inmediatamente perceptible por sus beneficiarios directos".

Con todo, en el parecer de la Comisión, se ligaba la cuestión de la naturaleza jurídica de la Carta a dos cuestiones previas que convendría resolver antes de pronunciarse sobre el particular: en primer lugar, a su propio contenido, pues sólo ante un texto suficientemente ambicioso tendría sentido tomar una decisión sobre su incorporación; y, en segundo lugar, en el caso de que finalmente se optara por la misma, a las cuestiones técnicas de diversa índole relacionadas con las reformas de los Tratados que deberían necesariamente tenerse en cuenta.

El proyecto de Carta fue finalmente terminado por la Convención y enviado al Consejo el 2 de octubre de 2000, para su debate y eventual aprobación durante la cumbre celebrada en Biarritz. Ante la posibilidad de que fuera entonces cuando se tomara alguna decisión sobre la naturaleza jurídica de la Carta, de nuevo las instituciones comunitarias consideraron oportuno pronunciarse sobre el particular. Así, el Parlamento instó de nuevo al Consejo a que iniciara los trámites para integrar la Carta en el TUE, de modo que en la cumbre de Niza se estuviera en condiciones de tomar una decisión al respecto. $Y$ de nuevo la Comisión emitió una comunicación, centrada esta vez sobre la naturaleza jurídica de la Carta, que elevaba ahora esta cuestión - una vez alcanzado el objetivo de un texto articulado coherente, preciso y ambicioso- al lugar central del debate.

En su nuevo comunicado, la Comisión, aun admitiendo que la Carta surtiría también plenos efectos jurídicos aun en el caso de no ser incorporada - cuestión ésta sobre la que volveremos más adelantereconocía, sin embargo, que "es poco probable que las expectativas suscitadas en la opinión pública por la decisión de elaborar la Carta puedan satisfacerse con una mera proclamación por las instituciones comunitarias, que no vaya seguida de la incorporación de la Carta a los Tratados", afirmando:

"(..) el destino de la Carta, por razón de su contenido, su rigurosa formulación jurídica y su considerable valor político y simbólico, es incorporarse tarde o temprano a los Tratados. Para la Comisión, tal incorporación no es, por consiguiente, una cuestión que haya de abordarse de una manera teórica o doctrinal, sino que, por el contrario, debe tratarse en términos de eficacia y buen sentido jurídicos. Por ello, es preferible, por razones de visibilidad y seguridad jurídica, que la Carta resulte vinculante por sí misma, y no a través de su 
interpretación jurisprudencial. En la práctica, la cuestión pertinente es la del momento y de las modalidades de su incorporación a los Tratados".

Biarritz, sin embargo, no supuso ninguna toma de postura por parte del Consejo, que se limitó a aprobar la Carta, solicitando igualmente la aprobación de la Comisión y del Parlamento, y posponiendo de nuevo la decisión final para la cumbre de diciembre. Antes de ésta, y con ocasión de su aprobación por el resto de las instituciones, hubo un nuevo posicionamiento del Parlamento, que, al mandatar a su Presidenta para que procediera, conjuntamente con el Presidente del Consejo $y$ el Presidente de la Comisión, a la proclamación solemne de la Carta en Niza, recordaba a estas instituciones su intención de debatir de nuevo sobre la cuestión de su naturaleza jurídica. En la sesión de 30 de noviembre, una semana antes de la cumbre, el pleno del Parlamento reiteró de nuevo su parecer de que la Carta debería incorporarse a los Tratados.

Por fin, el 7 de diciembre, como se sabe, el Consejo, la Comisión y el Parlamento proclamaron la Carta, en una ceremonia solemne oscurecida en los medios de comunicación por la resonancia que alcanzaron las manifestaciones en contra de la globalización que tuvieron lugar coincidiendo con la cumbre europea y a pocos metros de la misma.

A lo largo de más un año de debate - debate que, por otra parte, no puede considerarse cerrado en la actualidad, pues las posibilidades de incorporación siguen abiertas en el futuro- las instituciones comunitarias, la academia y la opinión pública polemizaron sobre el carácter que habría de tener la Carta. Las opiniones, dictámenes y resoluciones emitidas al respecto versan, sin embargo, a veces de modo simultáneo, sobre tres cuestiones que, aunque relacionadas entre sí, no son, ni mucho menos, idénticas: en primer lugar, su naturaleza jurídica, en segundo lugar, su carácter vinculante y, en tercer lugar, su incorporación a los Tratados. Conviene ahora, por lo tanto, hacer algunas precisiones al respecto.

\section{LA NATURALEZA JURIDICA}

Tanto si la Carta llega a tener fuerza vinculante como si no, y con independencia de que ésta se alcance mediante su incorporación a los Tratados o mediante otras vías, hay una nota esencial de la misma (la que, probablemente, de modo más directo condiciona en esencia su auténtica naturaleza jurídica) que permanecerá inalterable: su carácter principial. 
En efecto, la Carta, como toda norma de alcance general sobre derechos fundamentales, consiste en una serie de disposiciones mucho más cercanas a la positivación de principios - aquéllos cuya protección se ha considerado de envergadura suficiente como para erigirlos en derechos fundamentales de los ciudadanos de la Unión-que al establecimiento de una colección de reglas claras y precisas. Muy probablemente, en consecuencia, la respuesta más certera a la cuestión de cuál sea la naturaleza jurídica de la Carta es que ésta es una codificación de aquellos principios generales del derecho comunitario cuyo respeto debe considerarse un derecho fundamental de sus ciudadanos.

La naturaleza, asi entendida, de la Carta, se refleja en gran parte de su articulado. Los derechos fundamentales a la libertad y a la seguridad (art. 6 CDFUE), a la libertad de conciencia y religión (art. 10 CDFUE) o a la libertad de expresión (art. 11 CDFUE) son buenos ejemplos de ello. ¿Puede añadir algo la incorporación a los Tratados de estas disposiciones? En principio, parece que poco, al menos con respecto al respeto y protección de estos derechos por parte de la Unión. Aun en el caso de que se incorporaran, no dejarían de ser fórmulas genéricas, principios, positivados como derechos, pero sin un perfil concreto, sin un establecimiento claro de un estándar de protección al que referirse. A este respecto, la Carta cumple, aun sin formar parte de los Tratados, una importante función al especificar cuáles son los derechos que deben considerarse fundamentales (incluyendo, por cierto, algunas novedades interesantes en su catálogo) y al establecer cláusulas ya conocidas por algunos ordenamientos internos, como la que ordena que cualquier limitación de los mismos respete su contenido esencial (art. 52 CDFUE). Es en este sentido en el que parece que al TC español le ha bastado la aprobación de la Carta para citarla, antes incluso de su proclamación, en algunas de sus sentencias.

Ahora bien, si puede aceptarse, por lo dicho, que la incorporación no cambiaría en gran medida, desde esta perspectiva, la naturaleza jurídica de la Carta, debe al mismo tiempo admitirse que sí tendría importantes consecuencias para la naturaleza jurídica de los derechos de la Carta. Más allá del juego de palabras, se quiere poner ahora el énfasis en una serie de efectos que la incorporación podría producir, por una parte, sobre las disposiciones de la Carta en las que, de un modo $u$ otro, se proclaman derechos, $y$, por otra, sobre aquéllas que -a diferencia de las que acaban de citarse en el párrafo anterior- establecen, además, un estándar preciso de protección o de prohibición con respecto al derecho que establecen. 
Por lo que hace a la primera cuestión, probablemente el problema mayor que traiga consigo la ausencia de incorporación sea la dificultad de una actuación del legislador comunitario jurídicamente adecuada a los derechos fundamentales que la Carta aspira a reconocer. Hace ya tiempo - desde la consolidación de la plena normatividad constitucional- que el constitucionalismo contemporáneo ha abandonado la visión del legislador como un peligro potencial para los derechos que consagran las Constituciones. Por el contrario, como magistralmente demostró Peter HäBERLE, los derechos exigen, hoy, de la colaboración internormativa entre constituyente y legislador, pues éste es, una vez que han sido establecidas por las Constituciones, el auténtico artífice de que puedan llevarse a la práctica en su plenitud. La Carta tiene, en este sentido, una función constitucional de prefiguración de los derechos y de establecimiento (deficiente, hay que admitir) de mecanismos de garantía. Pero estos derechos prefigurados por la Carta tienen que ser configurados por el legislador, como por otra parte la propia Carta reconoce en buena parte de su articulado, hasta el punto de que la falta de actuación del legislador como configurador de los derechos puede quebrar el necesario equilibrio estructural entre Consitución, legislación y jurisdicción ( $F$. BaLAGUER), obligando a los órganos jurisdiccionales a recurrir, como únicos parámetros de control de sus posibles limitaciones, a los principios de razonabilidad y proporcionalidad.

Pues bien, la falta de incorporación a los tratados impedirá, con independencia de los efectos que pueda también producir en el mismo sentido sobre el legislador nacional, que el legislador comunitario pueda regular los derechos que la Carta establece actuando como legislador de los derechos fundamentales, es decir, respetando una serie de principios y garantías estructurales a la hora de acometer su desarrollo. Es claro que, no estando la Carta incorporada al derecho originario, el legislador comunitario acometerá la regulación de los derechos que en ella se contemplan con base en el fundamento jurídico que, en cada caso, establezca el Tratado, pero sin que estructural ni procedimentalmente pueda hablarse de un legislador de los derechos fundamentales. Del mismo modo, la no incorporación dificultará la consolidación dogmática en el derecho comunitario de esta categoría, iniciada tímidamente con la introducción, tras la aprobación del TUE, de las disposiciones sobre la ciudadanía.

La segunda cuestión nos remite a las disposiciones de la Carta que sí contienen reglas precisas, como cuando se prohibe la pena de muerte (art. 2 CDFUE) o cuando se incluye en el derecho a la vida fami- 
liar y profesional «el derecho a un permiso pagado por maternidad y a un permiso parental con motivo del nacimiento o de la adopción de un niño" (art. 33.2 CDFUE). Discernir si el respeto a estas reglas es o no, $y$ en qué grado, obligatorio para la Unión, nos lleva a tratar la cuestión del efecto vinculante de la Carta.

\section{EL EFECTO VINCULANTE}

¿Cuál es el efecto vinculante de la Carta en la situación actual, es decir, sin que la misma se haya incorporado formalmente al derecho originario? Cabe afirmar que, con las salvedades que acaban de precisarse en torno al modo en el que la naturaleza jurídica de los derechos consagrados en la Carta se vería afectada por una eventual incorporación, el efecto vinculante de la misma seguiría siendo, en gran medida, similar. Como se ha dicho, la Carta está llamada a ser la positivación de los principios generales del derecho comunitario a los que hasta ahora se ha referido la jurisprudencia del TJCE. Su función principal, tanto si se incorpora a los Tratados como si no, es dotar a la Unión de un catálogo preciso de cuáles son estos principios generales, $y$, en algunas ocasiones, establecer también, mediante mandatos o prohibiciones, estándares precisos de protección de los mismos. Desde este punto de vista, su respeto por parte de los operadores jurídicos comunitarios no parece que vaya a depender en gran medida de su incorporación formal a los Tratados.

Podemos ilustrar lo anterior con algunos ejemplos sacados de la actividad de las instituciones comunitarias. Así, la Comisión está considerando expresamente la Carta y el respeto a los derechos que en ella se establecen en las propuestas de normas comunitarias que se han elaborado a partir de su proclamación. En su propuesta de directiva relativa al estatuto de los nacionales de terceros países residentes de larga duración, de mayo de este año, por ejemplo, la Carta es profusamente citada, no sólo en los considerandos del preámbulo o exposición de motivos de la directiva, sino también a lo largo de su articulado, en el que se especifica que determinadas reglas establecidas por la directiva deben interpretarse según lo dispuesto por los correspondientes artículos de la Carta, positivando así una suerte de interpretación conforme del derecho derivado con respecto a lo establecido en la misma (que, por otra parte, sugiere una especie de incorporación por partes). Es significativa también, a este respecto, la acción emprendida por el Defensor del Pueblo Europeo solicitando a las instituciones comunitarias informes detallados sobre su política de reclutamiento de 
personal en relación con la existencia de una posible discriminación por edad, acción que se basa expresamente en lo señalado por el art. 14 CDFUE. Tomemos, en fin, un tercer e igualmente significativo ejemplo: la Carta, como parece desprenderse de los informes que al respecto emite la Comisión, ha adquirido un papel importante en la definición de la política exterior de la Unión en relación con la exigencia de respeto de la democracia y los Derechos Humanos.

De modo que la Carta parece destinada, a pesar de su no incorporación a los Tratados, a vincular a las instituciones comunitarias en el desarrollo de sus funciones. Ahora bien, para que podamos calificar esa vinculación como una obligación jurídica, no es suficiente que se dé, en la práctica, este respeto, sino que también es necesario, como es claro, que pueda obligarse al mismo cuando las instituciones lo han desatendido o ignorado. En este sentido, a nadie se le escapa que la posibilidad de que la Carta llegue a considerarse un texto vinculante depende, sobre todo, del Tribunal de Justicia de la Comunidad.

El propio texto de la Carta ofrece, de una manera en cierto modo paradójica, algunas potencialidades en este sentido. Sabido es que la Carta se encuentra plagada de cautelas sobre su propio perfil en el seno del derecho comunitario, cautelas que se han traducido en disposiciones, tanto en su preámbulo como en su articulado, que establecen claramente que la Carta no supone nuevos objetivos o misiones para la Comunidad, y que los derechos que en ella se consagran no son sino manifestaciones de otros derechos previamente reconocidos en el ordenamiento comunitario, bien de modo expreso, en diversas disposiciones repartidas por los Tratados y el derecho derivado, bien de modo implícito, en la jurisprudencia del TJCE a través de los principios generales del derecho. Sin duda alguna, estas cautelas que la propia Carta incluye estaban encaminadas a acallar las críticas que la misma podría suscitar desde el punto de vista del principio de subsidiariedad o del de competencias tasadas, o las que podrian deducirse del dictamen 2/94 del TJCE sobre la ausencia de un título genérico en base al cual la Comunidad pudiera legislar sobre derechos fundamentales.

Sin embargo, estas mismas disposiciones pueden producir un efecto en cierto modo contrario al pretendido, en un nuevo y significativo ejemplo de la autonomía de la que pueden gozar las normas jurídicas una vez que son aprobadas por el legislador, pues si admitimos como cierto que la Carta no añade nada a lo que ya existía en el ordenamiento comunitario, y que sus disposiciones protegen derechos que ya estaban previamente reconocidos en este ordenamiento, entonces 
será difícil negarle la fuerza jurídica propia de las normas comunitarias en las que éstas se fundamentan. La Carta no vendría sino a positivar un catálogo de derechos que ya previamente habrían sido objeto de un reconocimiento disperso o implícito a través de diversas vías, $y$, en esa medida, sus normas tendrian la fuerza jurídica de aquéllas en las que estos derechos se contendrían. Si, como hemos visto en el epígrafe anterior, donde la Carta establece principios, su incorporación a los Tratados sería en gran medida irrelevante, donde establece estándares precisos de protección sería, ahora, innecesaria.

Esta interpretación ha sido ya acogida en el ámbito del TJCE, si bien, hasta el presente, sólo en las conclusiones de los abogados generales. Así, en el asunto 173/99, el abogado general Tizzano empleó un argumento muy parecido al que acaba de mencionarse - que en realidad se basa en sus conclusiones-para postular que el derecho a vacaciones anuales retribuidas de los trabajadores debía considerarse un derecho fundamental del ordenamiento comunitario, citando el preámbulo de la Carta para justificar que su artículo 31.2 - donde se recoge - constituía "la confirmación más cualificada y definitiva" de su naturaleza como tal. Este tipo de argumento ha sido también aplicado por el abogado general JACOBS en las conclusiones del asunto C-270/99, en el que se aplica el artículo 41.1 CDFUE, que establece el derecho a que las instituciones y órganos de la Unión traten los asuntos que se les planteen uimparcial y equitativamente $y$ dentro de un plazo razonable», si bien reconociendo que la Carta "no es obligatoria jurídicamente en sí misman.

Hasta ahora, sin embargo, la argumentación que más directamente se ha basado en la Carta para controlar la validez del derecho comunitario se encuentra en las conclusiones - de nuevo del abogado general JACOBS - del asunto $\mathrm{C}-377 / 98$, relativas al recurso de anulación presentado por los Países Bajos contra la Directiva del Parlamento Europeo y del Consejo de 6 de julio de 1998 que regula las invenciones biotecnológicas, una materia sobre la que la Carta, especialmente en su art. 3, se ha mostrado particularmente sensible.

Las alegaciones contra la directiva se centraban, entre otros aspectos, en la posible vulneración de derechos fundamentales que podría deducirse de su artículo 5, que, aunque establece en su apartado primero que no pueden constituir invenciones susceptibles de ser patentadas ni el "cuerpo humano en los diferentes estadios de su constitución y de su desarrollo", ni "el simple descubrimiento de uno de sus elementos, incluida la secuencia o la secuencia parcial de un gen", sí permite, en su apartado segundo, patentar elementos del cuerpo 
humano que se hubieran "obtenido de otro modo mediante un procedimiento técnico, incluida la secuencia o la secuencia parcial de un gen", y ello "aun en el caso de que la estructura de dicho elemento sea idéntica a la de un elemento natural».

Para los Países Bajos, a cuyas alegaciones se sumó Italia, la posibilidad de realizar estas patentes atentaba contra el derecho fundamental a la dignidad humana. Además, imputaba a la directiva, como otras posibles violaciones de derechos fundamentales, su falta de previsión sobre la información que debía darse al paciente receptor de materia tratada mediante biotecnología, pues la directiva regula sólo la información que debe recibir el donante, $y$, con respecto a éste, que no estableciera su derecho, como donante de elementos aislados del cuerpo humano patentados, a tener, como mínimo, un cierto control sobre el destino de su donación. Los recurrentes alegaron, por esta razón, una vulneración del respeto debido a la vida privada, al secreto médico, a la integridad física y a la información personal, todos ellos entendidos como derechos fundamentales.

Las alegaciones contra la directiva, si bien no mencionan la CDFUE, pues fueron formuladas con bastante antelación a su proclamación, encuentran en ésta un importante tratamiento, ya que la Carta, por una parte, ha consagrado el respeto a la dignidad humana como un derecho fundamental autónomo (art. 1 CDFUE), $y$, por otra, ha establecido también (art. 3 CDFUE), en relación con el derecho a la integridad física y psíquica, una serie de derechos fundamentales en el marco de la medicina y la biología, entre ellos, el del consentimiento libre e informado, la prohibición de prácticas eugenésicas o que tengan por finalidad la selección de las personas, la prohibición de convertir en objeto de lucro el cuerpo humano o partes del mismo y la prohibición de la clonación reproductora de seres humanos. De todo ello dio buena cuenta el abogado general en sus conclusiones, admitiendo que

"(..) no hay duda de que los derechos invocados por los Países Bajos son efectivamente derechos fundamentales, cuyo respeto debe garantizarse por el ordenamiento jurídico comunitario. El más fundamental de estos derechos quizá sea el derecho a la dignidad humana, que se consagra en la actualidad en el artículo 1 de la Carta de los derechos fundamentales de la Unión Europea, donde se establece que la dignidad humana es inviolable y será respetada y protegida. El derecho de los donantes de elementos del cuerpo humano y de los receptores de tratamientos médicos a prestar su consentimiento libremente y con conocimiento de causa puede también considerarse fundamental. Este derecho se refleja igualmente en el artículo 3, apartado 2, de la Carta de derechos fundamentales 
de la Unión Europea, a tenor del cual en el marco de la medicina y la biología se respetará "el consentimiento libre e informado de la persona de que se trate, de acuerdo con las modalidades establecidas en la leym"

La conclusión del abogado general al respecto es clara: "hay que reconocer que cualquier instrumento comunitario que vulnere tales derechos es ilegaln.

Forzoso es reconocer, con todo, que en la única sentencia que hasta ahora conocemos de los asuntos citados, la C-173/99, el Tribunal ha preferido obviar cualquier referencia directa a la Carta para resolver la cuestión planteada, citando en cambio, para fundamentar su decisión, lo establecido en la Carta comunitaria de derechos sociales fundamentales de los trabajadores. Sabido es, sin embargo, que no siempre el Tribunal acoge con rapidez los postulados, generalmente más avanzados y no infrecuentemente mejor elaborados (aunque sólo sea por no tener que plegarse a las exigencias de una decisión colegiada donde no pueden hacerse pública las posibles disidencias), de los abogados generales, siendo también cierto, por otra parte, y como es obvio, que puede no llegar a producirse nunca.

\section{LA INCOPORACIÓN A LOS TRATADOS}

La naturaleza jurídica de la Carta —no así la de los derechos que consagra - y su posible efecto vinculante son, pues, relativamente independientes de que su texto se incorpore a los Tratados. Esta posibilidad - la de la incorporación- sigue sin embargo aún abierta, pues la proclamación de la misma llevada a cabo en la cumbre de Niza no significó una opción definitiva en sentido contrario. Es cierto que la decisión de posponer la incorporación se debió, sobre todo, a la inexistencia de un acuerdo generalizado entre los Estados miembros sobre el valor que debía concederse a la Carta. Pero lo es también que la incorporación exigía, desde el punto de vista técnico, una serie de requisitos que difícilmente se podrían haber abordado durante la cumbre. En efecto, en el caso de incorporar la Carta a los Tratados, probablemente al TUE, como pedía el Parlamento, habría que haber modificado tanto éste como el resto de los tratados originarios. Estas reformas tendrían que haber actualizado algunos artículos ya existentes (por ejemplo, el mandato de respeto a los derechos fundamentales del artículo 6.2 TUE), mientras que otros habrían tenido que ser directamente suprimidos al encontrarse reproducidos ahora en el articulado 
de la Carta léste es el caso de las disposiciones sobre la ciudadanía europea de los arts. 17-22 TCE, ahora reproducidas en los arts 39-46 CDFUE). En consecuencia, habria que haber decidido si incorporar la Carta como tal -la decisión en todo caso aconsejable si se quería respetar la opción por la visibilidad-o modificar éstos y otros artículos de los Tratados en función de su contenido.

Además, la incorporación, en el caso de producirse, tendrá que llevarse a cabo teniendo en cuenta otras decisiones importantes sobre la protección de los Derechos Humanos en el seno de la Unión y sobre la misma estructura del derecho originario. En primer lugar, la opción por ratificar o no la Convención Europea de Derechos Humanos, un asunto aún pendiente tras las exigencias del dictamen 2/94 del TJCE y sobre el que también se han pronunciado las instituciones comunitarias. $Y$, junto a esto, los trabajos en curso de cara a una simplificación del derecho originario y la eventual sustitución de la compleja arquitectura actual que presentan los tratados por un Tratado Fundamental de la Unión, en el que la Carta ocuparía el lugar reservado a la parte dogmática de los textos constitucionales.

No cabe duda, pues, que la opción por la incorporación exigía haber tomado también una serie de decisiones políticas y técnicas que la cumbre de Niza no estaba en condiciones de tomar. Para que la misma se produjera no era sólo necesario que la Carta se redactara pensando como si la misma fuera a producirse, sino también que los Tratados fueran modificados para hacer la recepción de la Carta posible. La cuestión es, ahora, si la Unión sigue decidida o no a trabajar en este sentido.

\section{UNA REFLEXION FINAL}

Conviene ahora recordar que la Comisión, que desde el primer momento postuló la incorporación de la Carta a los Tratados, reconocía sin embargo que

"se puede apostar - sin mucho riesgo- que la Carta producirá sus efectos, también en el plano jurídico, sea cual fuere la naturaleza que se le atribuya (..) es evidente el Consejo y la Comisión, que están llamados a proclamarla solemnemente, difícilmente podrán ignorar en el futuro, cuando actúen como legisladores, un texto que ha sido preparado a petición del Consejo Europeo por todas las fuentes de legitimidad nacionales y europeas reunidas en un mismo foro. Del mismo modo, parece lógico que, a su vez, el Tribunal de 
Justicia se inspire en la Carta, como ya lo hiciera con otro textos sobre derechos fundamentales. Se puede lógicamente esperar que la Carta, en cualquier hipótesis, resulte vinculante a través de su interpretación por el Tribunal de Justicia como principios generales del Derecho Comunitarion.

De acuerdo con estas afirmaciones, sólo desde un formalismo exagerado puede negarse completamente la posibilidad de que la CDFUE, aun sin incorporarse a los Tratados, y habida cuenta de su naturaleza de Declaración de Derechos, pueda llegar a adquirir en el futuro próximo valor jurídicamente vinculante. Es cierto que la Carta no puede encuadrarse en ninguna de las fuentes típicas del derecho comunitario, pero no lo es menos que el propio ordenamiento comunitario no ha dejado de resistirse desde su aparición a una calificación típica. $Y$ no faltan ejemplos en la historia jurídica contemporánea, como han puesto de relieve estudios hoy ya clásicos, como el de STEIN, dentro del propio derecho comunitario, o el de CORTNER, sobre la Bill of Rights norteamericana, de normas que llegaron a adquirir un valor distinto del que le correspondía por su posición en el sistema formal de fuentes: el que les deparó, con el tiempo, el diálogo (de nuevo HäBERLE) entre sus destinatarios y sus aplicadores.

\section{FUENTES Y REFERENCIAS}

El texto oficial de la Carta de Derechos Fundamentales de la Unión Europea, proclamado solemnemente en Niza el 7 de diciembre de 2000 por los presidentes del Consejo, de la Comisión y del Parlamento Europeo, se publicó en el Diario Oficial de las Comunidades Europeas (DOCE), serie C, n. 346, de 18 de diciembre de 2000; el mandato del Consejo Europeo de Colonia, la composición de la Convención decidida en la cumbre de Tampere y todos los documentos relacionados con el proceso de elaboración de la Carta se encuentran en la red, en la dirección habilitada al efecto por el Consejo, http://db.consilium.eu.int/df. Una introducción general a los problemas que plantea la CDFUE puede verse, en español, en Ricardo ALONSO, "La Carta de los derechos fundamentales de la Unión Europea», en Gaceta Jurídica de la Unión Europea 209 (2000), pp. 3-17

Los informes académicos más importantes que precedieron a la aprobación de la Carta fueron el informe Alston-WeILeR [Philip ALSTON y Joseph WEILER, The European Union and Human Rights: Final Project Report on an Agenda for the Year 2000, Florence, 
European University Institute, 1998] y el infome Simitis [SIMITIS, S.; Bell, C.; Betten, L.; Frowein, J.A.; Koskinen, P.K.; Martín Retortillo. L.; Pizzorusso, A. y Rosetto, J., Afirmación de los derechos fundamentales en la Unión Europea: ha llegado el momento de actuar, Comisión Europea, Bruselas, 1999]. Algunos de estos informes insistían (probablemente para evitar los inconvenientes que podrian plantear principios comunitarios como el de subsidiariedad o el de competencias tasadas) en que para desarrollar un política adecuada de protección de los derechos fundamentales en la Unión no era necesario enmendar los Tratados, sino tan sólo tomar medidas políticas y de carácter organizativo, pero en otros se postulaba claramente, por el contrario, acometer la elaboración de un texto articulado en el que se enumeraran los derechos a proteger, texto que debería después introducirse en una parte especial o en un título particular de los Tratados, y ello no sólo en aras de la obligatoriedad jurídica del mismo, sino también para dotar a la decisión de proteger los derechos fundamentales al más alto nivel de una apropiada "visibilidad", una de las preocupaciones fundamentales que luego haría suya la Convención redactora de la Carta.

La decisión de la Convención redactora de elaborar la Carta "como si" fuera a incorporarse a los Tratados, puede verse en el Informe del vicepresidente de la Convención, M. BraBANT, presentado a la asamblea de la misma el 26 de septiembre de 2000, reproducido en doc. CHARTE 4958/00, en www.db.consilium.eu.int/df, pp 2 y ss. Las resoluciones de otras instituciones comunitarias que se han citado en el texto son las siguientes: del Parlamento, Resolución del Parlamento Europeo sobre la elaboración de una Carta de los Derechos Fundamentales de la Unión Europea (C5-0058/1999-1999/2064 (COS)), de 16 de marzo de 2001; Resolución del Parlamento Europeo sobre la Carta de los Derechos Fundamentales de la Unión Europea, B50767/2000, de 3 de octubre de 2000; y Decisión del Parlamento Europeo sobre la aprobación del proyecto de Carta de los Derechos Fundamentales de la Unión Europea (C5-0570/2000), de 14 de noviembre de 2000; la referencia a la sesión parlamentaria de 30 de noviembre puede verse en el Boletín de la Unión Europea, no 11/2000. De la Comisión, la Comunicación sobre la Carta de los Derechos Fundamentales de la Unión Europea de 13 de septiembre de 2000, COM(2000) 559, y la Comunicación de la Comisión al Parlamento Europeo, al Consejo, al Comité Económico y Social y al Comité de las Regiones sobre la naturaleza de la Carta de los Derechos Fundamentales de la Unión Europea, $\operatorname{COM}(2000) 644$; del Comité de las Regiones, la Resolución sobre el proyecto de Carta de Derechos 
Fundamentales de la Unión Europea de 20 de septiembre de 2000 (CDR 140/2000; y del Comité Económico y Social, la Opinión, de la misma fecha que la anterior resolución, "Hacia una Carta de Derechos Fundamentales de la Unión European, SOC/013.

Se ha citado también en el texto la Propuesta de Directiva del Consejo relativa al estatuto de los nacionales de terceros países residentes de larga duración (presentada por la Comisión), Documento 501PC0127, de 21 de mayo de 2001; aparte de en el Preámbulo, la propuesta de directiva se refiere a la Carta, del modo que se explica en el texto, en los artículos 4, 13 y 15 . La referencia a la acción del Defensor del Pueblo Europeo se ha tomado de The European Ombudsman Press Release No. 10/2001, de 14 de mayo de 2001.En fin, la referencia del informe que se cita como ejemplo de la relevancia de la Carta en la acción exterior de la Comunidad es Commission StafF Working Document, Report on the implementation of the European Initiative for Democracy and Human Rights in 2000, de 22 de mayo de 2001, SEC(2001) 801.

En cuanto a la jurisprudencia y otras resoluciones, se cita en el texto, por este orden, la STC 290/2000, sobre la Ley Orgánica 5/1992, de 29 de octubre, de Regulación del Tratamiento Automatizado de los Datos de Carácter Personal y la STC 292/2000, sobre su sucesora, la Ley Orgánica 15/1999, de 13 de diciembre, de Protección de Datos de Carácter Personal. Ambas (la primera de ellas en el voto particular del Magistrado Manuel Jimenez de PARGA), citan la CDFUE; el Dictamen del TJCE 2/94, sobre la Adhesión de la Comunidad a la Convención Europea de Derechos Humanos, de 16 de marzo de 1996; las Conclusiones del Abogado General en los asuntos C-173/99, Broadcasting, Entertainment, Cinematographic and Theatre Union (BECTU) contra Secretary of State for Trade and Industry, de 8 de febrero de 2001 (TIZZANo); C-270-99, Z. Contra Parlamento Europeo, de 22 de marzo de 2001 (JaCOBS), y C-377-98, Reino de los Países Bajos contra Parlamento Europeo y Consejo de la Unión Europea, de 14 de junio de 2001 (JACOBS). Finalmente, la STJCE C-173/99, Broadcasting, Entertainment, Cinematographic and Theatre Union (BECTU) contra Secretary of State for Trade and Industry, de 26 de junio de 2001.

Las referencias de autores que aparecen en el texto se refieren, a las siguientes obras: de Peter Häвerle, sus contribuciones en Antonio LÓPEZ PINA [ed] La garantía constitucional de los derechos fundamentales. Alemania, España, Francia e Italia, Madrid, Civitas, 1991, y su "¿Existe un espacio público europeo?", en Revista de Derecho Comunitario Europeo 3 (1998) pp. 113-36; de Francisco BALAGUER, 
"Niveles y técnicas internacionales e internas de realización de los derechos en Europa. Una perspectiva constitucional", en Nuove frontiere dei diritti fondamentali, Torino Giapichelli, 2000; de Peter STEIN, "Lawyers, judges and the making of a transnational constitution", en American Journal of International Law 75 (1981), pp. 1-27; y de Richard CORTNER, The Supreme Court and the second Bill of Rights. The fourteenh Amendment and the Nationalization of Civil Liberties, Madison, Univeristy of Wisconsin Press, 1981.

Permítaseme, finalmente, y para un analisis general sobre la Carta, en el que se estudian también aspectos que no se han tratado en el texto, remitirme a mi trabajo, Ángel RodRiguez, Integración Europea $y$ Derechos Fundamentales, Madrid, Civitas, 2001, especialmente pp. 203 $y$ ss. 